\title{
ON REGULARIZATION SCHEME DEPENDENCE OF PREDICTIONS IN INFLATIONARY COSMOLOGY
}

\author{
Andrei Linde and Arthur Mezhlumian \\ Department of Physics, Stanford University, Stanford, CA 94305, USA
}

(October 8, 2018)

\begin{abstract}
We show that there exists a large class of regularization schemes for probabilistic predictions in the theory of a self-reproducing inflationary univere, all of which eliminate the apparent dependence on the time reparametrization. However, all these schemes lead to different answers for relative probabilities of finding various types of post-inflationary universes. Besides, all these schemes fail to be reparametrization invariant beyond the range of the inflaton field close to end of inflation boundary. Therefore, we argue that at the current level of understanding, the simple regularization schemes associated with cutoffs at equal time hypersurfaces are as good as the recently proposed more complicated procedures which try to fix the time-reparametrization dependence.
\end{abstract}

PACS: 98.80.Cq, 98.80.Hw

SU-ITP-95-24, gr-qc/9511058

One of the most unusual, but quite general features of inflationary models [1] is the self-reproduction and eternal expansion of the universe. This effect has especially dramatic consequences in the context of chaotic inflation [2], but in fact it is very general and occurs in new inflation as well [3]. For a wide range of initial conditions, the universe enters a stage of stationary evolution which never ends, thus making the very question of the original state almost irrelevant [4].

The volume of the universe in such scenarios grows without end. In particular, the volume of regions of the universe where inflation ends and life becomes possible also grows without end. In many models of inflation there exist several different minima of the effective potential which may correspond to drastically different types of post-inflationary physics: different values of constants of Nature, different symmetry properties, and even different dimensionality [1]. The number of different minima may be finite, but sometimes we encounter the situations where there is a continuous spectrum of all possible outcomes. A simplest example is given by the inflationary Brans-Dicke cosmology, where the effective gravitational constant after inflation may take all possible values from 0 to $\infty$ in different exponentially large parts of the universe [5]. In such a situation the knowledge of the fundamental Lagrangian by itself does not help us to explain the properties of the part of the universe where we live, and one should invent a more elaborate approach to explain observed properties of our universe or to predict the result of the future observations.

There are various levels of ambition which may be taken when trying to solve this problem. One may simply be satisfied by the fact that one of the minima of the effective potential corresponds to the low-energy physics of our type, invoking the weak anthropic principle [6] in order to explain why do we live in this particular type of minimum rather than any other. In this approach we answer the question whether it is possible to find ourselves in a local part of the universe such as we observe around us, not attempting to quantify how probable it is to find ourselves in there. This by itself is a very significant progress. Indeed, eternal reproduction of the universe in all its possible forms for the first time provides a real justification for the use of the weak anthropic principle [1, 4 ,7].

On the other hand, one may try to go even further and calculate relative probabilities associated with various states of the universe in order to prove that the state in which we happen to live is at least not very improbable - hoping to establish that we are in some sense typical. In the absence of better understanding of the origins of life, we may assume that the total number of observers living in domains of the universe with some specific properties is proportional to the total volume of such domains. Thus we presume that life and observers do spontaneously originate if there is enough space and if environment supports the life of that particular type. Another assumption is that we are typical observers, and therefore live in those places where most other observers live. (We will discuss the validity of these assumptions in the end of the paper.) Then by finding the properties of the parts of the universe where typical observers live one may try to explain some properties of our own part of the universe.

This more advanced version of anthropic principle

\footnotetext{
${ }^{*}$ We should emphasize that the problem of choice between different vacua appears in a purely physical context and therefore it cannot be ignored even by those opposed to anthropic considerations for philosophical reasons.
} 
was extensively used in inflationary cosmology for many years. For example, using this approach it was possible to justify validity of the first version of the Affleck-Dine mechanism of baryogenesis in the context of inflationary cosmology 8]. It was possible to show that the standard constraints on the axion mass $\left(m_{a} \gtrsim 10^{-5} \mathrm{eV}\right)$ disappear in inflationary cosmology if inflation ends with the Hubble constant $H<10^{9} \mathrm{GeV}$ (e.g. in hybrid inflation) [9]. Several attempts have been made to obtain strong anthropic constraints on the cosmological constant in the context of inflationary cosmology [10 13], to find the most probable value of the gravitational constant and of the parameter $\omega$ in the Brans-Dicke cosmology [5, 114, etc.

The difference between the two versions of anthropic principle is not clear cut. Although the weak anthropic principle does not require calculation of the relative probability of observing the local domain of the universe of our type as compared to domains of all other types, if such probability turns out to be infinitesimally small it would be difficult to justify the applicability of that principle. Thus, the results of the second approach may invalidate the results of the first one in some extreme cases. In general, one may consider the second approach as an attempt of a more quantitative use of the weak anthropic principle. Recently it was suggested to call this version of anthropic principle "the principle of mediocrity" [11, which emphasizes the assumption that we are typical. We will use a more conventional terminology for the reason to be discussed in the end of this paper.

Calculation of probabilities ignoring self-reproduction of the universe is a rather difficult but well defined task. Meanwhile, in an eternally self-reproducing universe this problem becomes much more complicated. Indeed, in this theory the universe looks like a growing fractal consisting of indefinitely large number of regions of all possible types. Since the total volume of the self-reproducing universe grows without end, the normalization of the probabilities associated with volumes containing matter in specific states becomes an ambiguous task. Some sort of regularization of infinitely large volumes should be made. If we try to compare volumes at different equal time hypersurfaces, the dependence on time parametrization proves to be very strong [4, 5,12 . One is forced to make a choice between different regularizations in order to make quantitative predictions based on relative probabilities.

The importance of this ambiguity is emphasized by the fact that it is not only the description of the global structure of the whole universe (far beyond the visible scale) which depends considerably on the choice of regularization. We found recently that many models of inflation predict, under a specific choice of regularization (namely, by making comparisons on the hypersurfaces of equal proper time), that the local part of universe which we observe should be spherically symmetric but may contain large radial inhomogeneity and we should be living not far from the geometrical center of our local patch [15, 16.
Depending on the choice of an inflationary model, this effect may be either insignificant and practically unobservable [17] or very pronounced [15]. Thus, the question of the dependence on time parametrization, or more generally, dependence on the regularization scheme in eternally inflating universe suddenly becomes related to observations in a quite direct way.

In a recent article [18] Vilenkin proposed a very interesting new approach to regularization of diverging probabilities in the context of eternal inflation. An important feature of his approach is that it apparently eliminates the dependence of the relative probabilities on the choice of the time parametrization. The remaining weak dependence is argued to be of the same order as the dependence on the operator ordering ambiguity in Wheeler-DeWitt equation, i. e. within the accuracy of the quantum cosmology approximation [19].

However, it is not quite clear whether the absence of strong dependence of the results on the choice of time parametrization is enough to justify the use of this regularization procedure. We will show in this article that, while retaining the general idea of Vilenkin's regularization prescription, it is possible to find many different implementations of this scheme. They do not coincide with the implementation proposed in 18, 19, and the answers which we get for relative probabilities are considerably different from the answers obtained there. Nevertheless, all those answers have the same time-reparametrization invariance features as the ones proposed by Vilenkin.

We will show that the stationarity of inflation leads effectively to implicit choice of time-parametrization in the regularization scheme proposed in [18,19]. Thus, the apparent independence on time parametrization comes at a price of dependence on regularization scheme. We will show also that this scheme has time-reparametrization invariance features for only part of the universe, namely for very low-energy regions near the end of inflation boundary and post-inflationary domains. The relative probabilities regarding the domains which are still in the process of active inflation do depend on time parametrization even in that approach. We will present some conclusions regarding the choices between various non-invariant answers and whether one has to insist on finding "better" invariant answers for the type of questions which we are interested in.

The evolution of the scalar field driving inflation (inflaton), coarse-grained over the spatial region of size $H^{-1}$ (called hereafter an $h$-region in short), in the slow rolling approximation in arbitrary time parametrization is described by the stochastic differential equation [20,21, (7):

$$
\begin{aligned}
\frac{d \phi}{d \tau} & =-\frac{V^{\prime}(\phi)}{3 H(\phi) T(\phi)}+\frac{H^{3 / 2}(\phi)}{2 \pi T^{1 / 2}(\phi)} \xi(\tau) \\
& \equiv v(\phi)+\sqrt{2 D(\phi)} \xi(\tau),
\end{aligned}
$$

where $\xi(\tau)$ is a gaussian white noise, $V(\phi)$ is the inflaton potential, and $H(\phi)=\sqrt{8 \pi V(\phi) / 3 M_{P}^{2}}$ is the Hubble pa- 
rameter during the slow roll. Different time parametrizations are related to the proper time by local path dependent transformation:

$$
t \rightarrow \tau(t)=\int^{t} d s T\left(\phi_{\xi}(s)\right)
$$

where $T(\phi)$ is a positive function, and its argument in (2) is a solution of (11) under $T \equiv 1$ (proper time) with a particular realization of the white noise. We also introduced compact notations (coinciding with those in 19] for their set $T(\phi)=H^{\alpha}(\phi)$ of time reparametrization functions) for the inflaton field dependent drift velocity

$$
v(\phi)=-\frac{V^{\prime}(\phi)}{3 H(\phi) T(\phi)},
$$

and diffusion coefficient

$$
D(\phi)=\frac{H^{3}(\phi)}{8 \pi^{2} T(\phi)} .
$$

There is an ambiguity in interpreting equation (11) (regardless of time variable chosen, even with $T \equiv 1$ which corresponds to proper time). One can interpret it as a stochastic differential equation in the sense of Ito or in the sense of Stratonovich. Without delving deep into these details, we just note that this ambiguity is related to operator ordering problem in Wheeler-DeWitt approach to quantum cosmology [4, 19, Since this source of ambiguity is not essential in our present analysis, we will choose for definiteness the multiplicative Stratonovich interpretation of (11) in this paper.

The local dynamics of inflaton field in the given $h$ region, described by (11), is accompanied by exponential expansion of the physical volume which may be regarded as a branching of $h$-regions into $e^{3}$ "daughter" domains within every interval $\Delta t \sim H^{-1}$ of proper time. Each such domain continues independent evolution according to (11) with its own realization of the white noise [4]. One can introduce various probability measures describing this process.

The simplest measure is the probability distribution of finding the value of inflaton $\phi$ at time $\tau$ in the given $h$-region disregarding any new domains generated during the inflation:

$$
P_{c}(\phi, \tau)=\left\langle\delta\left(\phi_{\xi}(\tau)-\phi\right)\right\rangle_{\xi},
$$

where $\phi_{\xi}(\tau)$ is the solution of (1) with a particular realization of the white noise $\xi(\tau)$, and the average is taken over all possible realizations of $\xi(\tau)$. This distribution satisfies the Fokker-Planck equation:

$$
\begin{aligned}
\frac{\partial}{\partial \tau} P_{c}(\phi, \tau) & =\frac{\partial}{\partial \phi}\left(D^{1 / 2}(\phi) \frac{\partial}{\partial \phi}\left(D^{1 / 2}(\phi) P_{c}(\phi, \tau)\right)\right) \\
& -\frac{\partial}{\partial \phi}\left(v(\phi) P_{c}(\phi, \tau)\right) .
\end{aligned}
$$

However, as we argued above, the comoving probability measure $P_{c}(\phi, \tau)$ is often not the best choice for calculating probabilities regarding typical observers of our type. A different measure, proportional to the physical volume generated during inflation

$$
\begin{aligned}
P_{p}(\phi, \tau) & =\left\langle\delta\left(\phi_{\xi}(\tau)-\phi\right) \operatorname{Volume}_{\xi}(\tau)\right\rangle_{\xi} \\
& =\left\langle\delta\left(\phi_{\xi}(\tau)-\phi\right) e^{3 \int_{0}^{\tau} \frac{H\left(\phi_{\xi}\left(\tau^{\prime}\right)\right)}{T\left(\phi_{\xi}\left(\tau^{\prime}\right)\right)} d \tau^{\prime}}\right\rangle_{\xi},
\end{aligned}
$$

was introduced in [21 23, 4 . Its evolution is described by an equation, which can be interpreted as a Fokker-Planck equation for branching diffusion 24, 迎:

$$
\begin{aligned}
\frac{\partial}{\partial \tau} P_{p}(\phi, \tau) & =\frac{\partial}{\partial \phi}\left(D^{1 / 2}(\phi) \frac{\partial}{\partial \phi}\left(D^{1 / 2}(\phi) P_{p}(\phi, \tau)\right)\right) \\
& -\frac{\partial}{\partial \phi}\left(v(\phi) P_{p}(\phi, \tau)\right)+n(\phi) P_{p}(\phi, \tau) .
\end{aligned}
$$

The intensity of branching is related to local rate of expansion of the universe [4]:

$$
n(\phi)=\frac{3 H(\phi)}{T(\phi)} .
$$

It was proven in [4] that the evolution of the eternal inflationary universe very soon approaches a stationary state, where all probability measures are dominated at late times by their ground state (fastest increasing or slowest decreasing) eigenfunctions:

$$
P(\phi, \tau)=\sum_{k=1}^{\infty} e^{\lambda_{k} \tau} \psi_{k}(\phi) \approx e^{\lambda_{1} \tau} \psi_{1}(\phi)
$$

Here $\psi_{k}(\phi)$ are the eigenfunctions of the diffusion generating operators on the right hand side of (6) or (8), respectively, and $\lambda_{k}$ are the corresponding eigenvalues, $\lambda_{1}>\lambda_{2}>\ldots>\lambda_{k}>\ldots$. We have estimated in [4] that this asymptotic regime establishes itself very quickly, within few thousands of Planck times.

Looking at equations (3) - (9) it becomes clear why most of the quantities calculated with the help of either $P_{c}$ or $P_{p}$ will depend on the choice of time parametrization (i. e. function $T(\phi)$ in our case). All parts of these equations explicitly depend on $T(\phi)$, therefore by taking a different functional form of $T(\phi)$ one will inevitably end up with quite different solutions $P_{c}$ and $P_{p}$.

In previous papers [4, 15] we argued that this dependence is an inherent feature of our problem since we are dealing with super-horizon scale quantities which need not be gauge-independent. As we mentioned before, the stationary solution of (8) increases with time indefinitely, so we need to perform a regularization to obtain relative probabilities. The simplest cut-off procedure which we implemented by taking the equal time hypersurfaces and comparing the volumes on such hypersurfaces, although 
clearly not unique, has the advantage of being directly related to the derivation of $(8)$ and the meaning of $P_{p}(\phi, \tau)$ which is the portion of the physical volume filled with inflaton at value $\phi$ on the time hypersurface $\tau$.

Moreover, it does not really make any difference if we count the integral of the three-dimensional volume from $\tau=0$ up to the time hypersurface $\tau$ or just count the volume in that hypersurface. Since the volume (and $P_{p}$ ) increases with time in the stationary regime as an exponent with positive constant coefficient $P_{p}(\phi, \tau) \sim$ $\psi_{1}^{(p)}(\phi) \exp \left(\lambda_{1}^{(p)} \tau\right)$, then the integral over time is dominated by its upper limit and is proportional (up to the pre-exponential constant factor $1 / \lambda_{1}^{(p)}$ ) to the integrand at the upper limit. Thus, the ratio of volumes or probabilities calculated in these two ways coincide with exponential accuracy.

The value of the constant $\lambda_{1}$ as well as the functional form of the normalized stationary probability distribution $\psi_{1}(\phi)$ depend crucially on the choice of the timeparametrization (functional form of $T(\phi)$ ). One of the few invariant features of the probability distribution is whether it is exponentially increasing or decreasing, or equivalently, whether there is a self-reproduction manifest in this measure or not. In branching diffusion terminology, the invariant feature is whether the branching process is super-critical or sub-critical. This is determined by the sign of $\lambda_{1}$, which does not change for most reasonable reparametrizations (unless we choose "geodesically incomplete" parametrization [4]25]).

The basic idea of Vilenkin's regularization scheme 18, 19 is to use the comoving measure $P_{c}$ for calculating the cut-off times, which are defined as times when all but a small portion of the comoving volume is already thermalized in the given minimum, and then to use those cut-off times to calculate the regularized ratios of volume weighted probabilities $P_{p}$. The reason why this works is that the comoving measure $P_{c}$ does not exhibit self-reproduction and its value decays with time exponentially with a negative constant coefficient $P_{c}(\phi, \tau) \sim \exp \left(\lambda_{1}^{(c)} \tau\right)$ due to the continuous outflow of probability into post-inflationary stage. In this measure any $h$-region slowly but steadfastly rolls down towards the values of inflaton field when the inflationary approximation breaks and the hot big bang begins. One can prove that $\lambda_{1}^{(c)}<0$ if there is any kind of boundary where inflationary regime ceases to exist. Therefore, the total probability $P_{c}$ of finding a domain still in inflationary stage converges at $t \rightarrow \infty$ and one can make a well defined statement on what portion of comoving volume is already in post-inflationary state.

However, from what is said above it is clear that there is nothing special about the comoving volume measure $P_{c}$ (besides the fact that it is well known and studied) - any measure which converges at $\tau \rightarrow \infty$ will be suited just as well for purposes of regularization. For example, one can consider a probability weighted with some measure not coinciding with volume. A particular case would be a probability density associated with branching diffusion process where branching intensity (in proper time) is just some arbitrary function $n(\phi) \neq 3 H(\phi)$ unrelated to the local rate of expansion of the universe. Since any branching intensity scales with time reparametrizations as $1 / T(\phi)$, the cancellation of the time parametrization dependence in the regularized volume ratio will still hold, as one can easily see from the final results belowif.

To be specific, we suggest the following, slightly more general measure to be used instead of $P_{c}$ in the cut-off procedure while retaining the general idea of [18, 19]:

$$
\begin{aligned}
P_{q}(\phi, \tau) & =\left\langle\delta\left(\phi_{\xi}(\tau)-\phi\right)\left(\text { Volume }_{\xi}(\tau)\right)^{q}\right\rangle_{\xi} \\
& =\left\langle\delta\left(\phi_{\xi}(\tau)-\phi\right) e^{3 q \int_{0}^{\tau} \frac{H\left(\phi_{\xi}\left(\tau^{\prime}\right)\right)}{T\left(\phi_{\xi}\left(\tau^{\prime}\right)\right)} d \tau^{\prime}}\right\rangle_{\xi} .
\end{aligned}
$$

This measure can be considered as appearing from multi-fractal analysis of the global structure of inflationary universe. Indeed, it has been established that the geometry of the eternal inflationary universe is fractal 21,26, [1]. However, different measures lead to different fractal dimensions. A natural way to interpret this structure is to consider the physical volume associated with every point as a multi-fractal measure defined on the underlying comoving base geometry. Then, the characterization (11) is very similar to the way the so called Renyi dimensions are introduced [27]. Physically speaking, this measure corresponds to taking into account only a fraction of newly generated volume at every time step. Taking $q=0$ we return to the case of $P_{c}$, while $q=1$ corresponds to $P_{p}$ (we will use superscript notations $c \leftrightarrow(q=0)$ and $p \leftrightarrow(q=1)$ for compactness and to retain compatibility with our notations in [4]) In general, one may consider $q<0$ as well.

This multi-fractal measure satisfies a branching diffusion equation, where the branching intensity is proportional to the old value (9) with a coefficient $q$ :

$$
\begin{aligned}
\frac{\partial}{\partial \tau} P_{q}(\phi, \tau) & =\frac{\partial}{\partial \phi}\left(D^{1 / 2}(\phi) \frac{\partial}{\partial \phi}\left(D^{1 / 2}(\phi) P_{q}(\phi, \tau)\right)\right) \\
& -\frac{\partial}{\partial \phi}\left(v(\phi) P_{q}(\phi, \tau)\right)+q n(\phi) P_{q}(\phi, \tau) .
\end{aligned}
$$

\footnotetext{
${ }^{\dagger}$ There is a particular choice of $n(\phi)$ which allows a simple interpretation. Let us take $n(\phi)=C / T(\phi)$, which becomes simply $n(\phi)=C$ for proper time. Then eq. (\&) describes the distribution of such observers who split producing a constant average number $C$ of new observers per unit proper time. For $C<0$, (8) describes observers who have a finite proper lifetime and, in average, die after $\Delta t \sim C^{-1}$.

${ }^{\ddagger}$ The correspondence with notations in [18, 19] is established using the following rules: their quantities without tilde correspond to our quantities with superscript $(c)$, the quantities with tilde correspond to our ones with superscript $(p)$, their $\gamma_{k}$ corresponds to our $\lambda_{k}$, while $\tilde{\gamma} \leftrightarrow \lambda_{1}^{(p)}$ and $\gamma \leftrightarrow-\lambda_{1}^{(c)}$.
} 
It is clear that for small enough values of $q$ the probability distribution $P_{q}$ (12) will be decaying with time just like $P_{c}$. Therefore, for small enough values of $q$ this measure can be used as an alternative regularization measure. Following the prescriptions in [18,19] we rewrite (12) in a probability flux form:

$$
\begin{aligned}
\frac{\partial}{\partial \tau} P_{q}(\phi, \tau) & =-\frac{\partial}{\partial \phi} J_{q}(\phi, \tau)+q n(\phi) P_{q}(\phi, \tau) \\
J_{q}(\phi, \tau) & =-D^{1 / 2}(\phi) \frac{\partial}{\partial \phi}\left(D^{1 / 2}(\phi) P_{q}(\phi, \tau)\right) \\
& +v(\phi) P_{q}(\phi, \tau) .
\end{aligned}
$$

Let there be two types of post-inflationary minima, denoted $\phi_{e i}^{(1)}$ and $\phi_{e i}^{(2)}$, respectively, where the subscript stands for "end of inflation". The total volume thermalized in these minima by time $\tau$ is given by the integral of the volume flux through the corresponding boundary:

$$
\mathcal{V}^{(1,2)}=\left|\int_{0}^{\tau} J_{p}\left(\phi_{e i}^{(1,2)}, \tau^{\prime}\right) d \tau^{\prime}\right|
$$

where the symbol $(1,2)$ is used for the first or the second minimum correspondingly.

The volumes $\mathcal{V}^{(1)}$ and $\mathcal{V}^{(2)}$ diverge at large $\tau$. Vilenkin's regularization prescription is to introduce cutoff times $\tau_{\epsilon}^{(1,2)}$ such that only a small portion of converging measure is still in the inflationary stage. We will use a general multi-fractal measure $P_{q}$ with small enough $q$ as such converging measure:

$$
\left|\int_{\tau_{\epsilon}^{(1,2)}}^{\infty} J_{q}\left(\phi_{e i}^{(1,2)}, \tau\right) d \tau\right|=\epsilon\left|\int_{0}^{\infty} J_{q}\left(\phi_{e i}^{(1,2)}, \tau\right) d \tau\right| \equiv \epsilon p^{(1,2)} .
$$

Here $p^{(1)}$ and $p^{(2)}$ are the total multi-fractal measures that will eventually thermalize in the first and second minima respectively. Then, the regularized expressions for total physical volume which will end up in the corresponding minima are given by:

$$
\mathcal{V}^{(1,2)}=\left|\int_{0}^{\tau_{\epsilon}^{(1,2)}} J_{p}\left(\phi_{e i}^{(1,2)}, \tau\right) d \tau\right|
$$

In order to estimate these integrals we have to find the flux (14) near the end of inflation boundary. Fortunately, it is a well known fact that below certain level of energy density one can neglect the diffusion (second derivative) part in (12) because it is much smaller than the drift (first derivative) part [1,21]. Therefore, within the applicability of this approximation in the stationary regime the flux is:

$$
J_{q}(\phi, \tau) \approx v(\phi) P_{q}(\phi, \tau) \approx v(\phi) \psi_{1}^{(q)}(\phi) e^{\lambda_{1}^{(q)} \tau}
$$

For small enough $\epsilon$ the characteristic cut-off times become very large and the use of stationary asymptotic (10) is justified. Indeed, the volume flux integral (17) is dominated by its upper limit, while the left hand side of (16) is dominated by its lower limit because of the signs of exponential coefficients $\lambda_{1}^{(p)}>0$ and $\lambda_{1}^{(q)}<0$ for sufficiently small $q$. As we mentioned before, for any $q$ (including $q=1) \lambda_{1}^{(q)}$ is the highest eigenvalue associated with the right hand side of (12):

$$
\begin{aligned}
\lambda_{1}^{(q)} \psi_{1}^{(q)}(\phi) & =\frac{d}{d \phi}\left(D^{1 / 2}(\phi) \frac{d}{d \phi}\left(D^{1 / 2}(\phi) \psi_{1}^{(q)}(\phi)\right)\right) \\
& -\frac{d}{d \phi}\left(v(\phi) \psi_{1}^{(q)}(\phi)\right)+q n(\phi) \psi_{1}^{(q)}(\phi) .
\end{aligned}
$$

Substituting (18) into (16) and (17), we get the cut-off times:

$$
\tau_{\epsilon}^{(1,2)}=\frac{1}{\left|\lambda_{1}^{(q)}\right|} \ln \left(\frac{\left|v\left(\phi_{e i}^{(1,2)}\right)\right| \psi_{1}^{(q)}\left(\phi_{e i}^{(1,2)}\right)}{\epsilon\left|\lambda_{1}^{(q)}\right| p^{(1,2)}}\right)
$$

and the regularized volumes:

$$
\mathcal{V}^{(1,2)}=\frac{1}{\lambda_{1}^{(p)}}\left|v\left(\phi_{e i}^{(1,2)}\right)\right| \psi_{1}^{(p)}\left(\phi_{e i}^{(1,2)}\right) e^{\lambda_{1}^{(p)} \tau_{\epsilon}^{(1,2)}} .
$$

From (20) and (21) we get the regularized ratio of volumes thermalized in the two minima:

$$
\begin{aligned}
r \equiv \frac{\mathcal{V}^{(2)}}{\mathcal{V}^{(1)}} & =\frac{\left|v\left(\phi_{e i}^{(2)}\right)\right| \psi_{1}^{(p)}\left(\phi_{e i}^{(2)}\right)}{\left|v\left(\phi_{e i}^{(1)}\right)\right| \psi_{1}^{(p)}\left(\phi_{e i}^{(1)}\right)} \\
& \times\left(\frac{\left|v\left(\phi_{e i}^{(2)}\right)\right| \psi_{1}^{(q)}\left(\phi_{e i}^{(2)}\right)}{\left|v\left(\phi_{e i}^{(1)}\right)\right| \psi_{1}^{(q)}\left(\phi_{e i}^{(1)}\right)} \frac{p^{(1)}}{p^{(2)}}\right)^{\frac{\lambda_{1}^{(p)}}{\left|\lambda_{1}^{(q)}\right|}} .
\end{aligned}
$$

This result is a generalization of equation (41) from article 19] for the multi-fractal measure $P_{q}$ used instead of $P_{c}$ for defining the cut-off procedure. Within the applicability of the no-diffusion approximation used to derive (22) one can easily solve (19):

$$
\psi_{1}^{(q)}(\phi)=\frac{C_{1}^{(q)}}{v(\phi)} \exp \left(\int_{\phi_{0}}^{\phi}\left[q \frac{n\left(\phi^{\prime}\right)}{v\left(\phi^{\prime}\right)}-\frac{\lambda_{1}^{(q)}}{v\left(\phi^{\prime}\right)}\right] d \phi^{\prime}\right) .
$$

We can see immediately that the dependence on the functional form $T(\phi)$ cancels out in the first term in the exponent, therefore it is time reparametrization invariant. The second term depends on $T(\phi)$, and therefore it is crucial that this term cancels in the formula for ratio of volumes (22). It indeed works out very nicely (remember that $\lambda_{1}^{(q)}<0$ for small $q$ ), and the final result after substituting (23) into (22) becomes: 


$$
r=C(q)\left(\frac{Z^{(2)}}{Z^{(1)}}\right)^{3}
$$

where the mildly varying coefficient $C(q) \sim 1$ is not important $\beta$, and the exponentially large volume factors are:

$$
\begin{aligned}
Z^{(1,2)} & =\exp \left\{\left(1+q \frac{\lambda_{1}^{(p)}}{\left|\lambda_{1}^{(q)}\right|}\right) \int_{\phi_{0}}^{\phi_{e i}^{(1,2)}} \frac{n(\phi)}{3 v(\phi)} d \phi\right\} \\
& =\exp \left\{-\left(1+q \frac{\lambda_{1}^{(p)}}{\left|\lambda_{1}^{(q)}\right|}\right) \int_{\phi_{0}}^{\phi_{e i}^{(1,2)}} \frac{4 \pi H(\phi)}{H^{\prime}(\phi)} d \phi\right\} .
\end{aligned}
$$

In the case $q=0$ this result coincides with the one obtained by Winitzki and Vilenkin [19], see their eq. (46). However, for small but not vanishing $q$, our result is exponentially different. As it is obvious from (25), our result has the same time reparametrization invariance features as its particular case in $q=0$, since it does not explicitly depend on the function $T(\phi)$, while the ratio of eigenvalues is stable with respect to time reparametrization, as it was shown in 19 . Therefore, we have proved that there exists a large family of regularization schemes, all of which satisfy the reparametrization invariance condition, but all lead to considerably different from each other answers.

Our results suggest that even nice features like time reparametrization invariance are not enough to uniquely determine the correct way of regularization of the quantities in quantum cosmology. It is not clear why would one of our multi-fractal regularization schemes be any better or worse than a particular scheme associated with $P_{c}$. One indeed has to have a much deeper than purely technical reasons in order to pick a particular regularization scheme in favor of many others.

Another important note regarding the reparametrization invariance of the regularized volume ratio is that its invariance is only partial. Indeed, the expression (24), and even the whole expression (22) are valid only sufficiently close to end of inflation boundaries, where the no-diffusion approximation holds. Beyond the region of its applicability, the explicit dependence on the functional form of $T(\phi)$, and therefore dependence on time reparametrizations will be unavoidable, since the more

\footnotetext{
$\S$ There is a subtle point here which is worth mentioning. The values of total multi-fractal measures $p^{(1)}$ and $p^{(2)}$ which will eventually thermalize in the two minima are not dependent on time parametrization. This is because regardless of which time variable we choose, the physics of the result remains the same for all converging measures: every given $h$-region will sooner or later be either in the first or in the second minimum. Therefore when calculating the total thermalized converging measure one will always get the same number.
}

complicated form of the complete solution of branching diffusion equation 4, 25 does not allow for magic cancellations like the one in (24).

This is even more important for chaotic inflation models most of which have a very narrow range of values of the inflaton field where the no-diffusion approximation is valid. For example, in the simplest such model based on the inflaton potential $\lambda \phi^{4} / 4$ the total range of the possible values of the inflaton field is $0 \lesssim \phi \lesssim \lambda^{-1 / 4} M_{\mathrm{P}} \sim 2 \times 10^{3} M_{\mathrm{P}}$ (here $\lambda \sim 10^{-13}$ is the value of the coupling constant obtained from constraints on CMB anisotropy). At the same time the range of applicability of the no-diffusion approximation is only $0<\phi<\lambda^{-1 / 8} M_{\mathrm{P}} \sim 40 M_{\mathrm{P}}$, i. e. narrower by almost two orders of magnitude $4,1,15]$.

Thus, in the simplest versions of chaotic inflation scenario the procedure suggested in [18,19] and its generalizations proposed above fail to produce reparametrization invariant results in the main part of the allowed values of the field $\phi$. In terms of energy density $\rho$, the results become reparamerization invariant only for $\rho$ about six orders of magnitude smaller than the Planck density! Of course, one could argue that predictions of quantum cosmology need not be reparametrization invariant during inflation since life cannot appear there anyway. However, this does not sound like a satisfactory answer if one want to maintain the consistency of this approach.

Despite the problems mentioned above, one should note that the regularization procedure suggested by Vilenkin has some advantages, and its results have a very simple physical interpretation: the ratio of volumes (24) in the case $q=0$ coincides with its value in the classical theory ignoring the process of self-reproduction. Indeed, the factor

$$
Z^{(1)}=\exp \left(-4 \pi \int_{\phi_{0}}^{\phi_{e i}^{(1)}} \frac{H(\phi)}{H^{\prime}(\phi)} d \phi\right)
$$

coincides exactly with the total expansion factor of the universe during the classical rolling of the field $\phi$ from $\phi_{0}$ to $\phi_{e i}^{(1)}$.

However, one may consider this result as being too simple: the central feature of our scenario, the effect of self-reproduction of the universe, has completely dropped out from the answer (for $q=0$ ). This is somewhat disturbing, but technically it is quite understandable since the regularization scheme of [18,19] is based on the investigation of the distribution $P_{c}$, which is not very suitable for study of the self-reproduction. This distribution correctly describes the typical fate of each individual observer which was present in the universe from the very beginning. However, there were no real observers during inflation, and the whole observable part of our universe was created from the place which was much smaller than the Planck length until the last 70 e-foldings of inflation. Thus, inhabitants of our part of the universe could not care less about the fate of imaginary observers 
living at the beginning of inflation. By focusing our attention on the portion of such "observers" entering the post-inflationary epoch we are ignoring the main portion of the volume of the universe which is produced in those rare domains which are jumping back towards large $V(\phi)$ in the process of self-reproduction of the universe.

Note also that all regularization procedures based on the use of the convergent measure $P_{q}$ have one feature in common. Since for any time parametrization the eigenvalues $\lambda_{1}^{(q)}$ are constants, it is evident from (20) and (21) that the regularization procedure considered in this paper implicitly favors a specific time parametrization, namely the parametrization in which time variable is the logarithm of the total volume (this variable was first introduced in the context of stochastic inflation in [20]). Indeed, the stationarity of inflation leads both the converging measure and diverging volume to be proportional to exponents of time variable with different coefficients. Therefore, cut-off times $\tau_{\epsilon}^{(1,2)}$ are proportional to logarithms of volume, making this choice of time parametrization implicitly preferred. In fact, it was argued in 18 that this time parametrization is indeed preferred, and the scale factor was the only variable to be used as clock during inflation. This is very similar to the argument used by Hawking when he was trying to prove that the arrow of time should turn back at the moment when the scale factor of a closed universe begins decreasing [28. The main problem with this argument is that the scale factor of the universe is the best clock only before the universe is observed. Then its wave function collapses into the wave function depending on the clock used by an observer, and after that the decrease of the scale factor does not lead to the reversal of the new time arrow [29. The natural clock used by observers of our type are based on oscillators rather than on the measurement of distances between galaxies.

Thus we need some additional reasons to pick up a particular regularization and use it to define a proper measure in quantum cosmology. Such reasons might not be completely determined by quantum field theoretical consistency of the description of eternally inflating universe, e.g. by time reparametrization invariance. One may need to add additional considerations regarding the type of questions which we are asking. In particular, one might want to take into account the preferences for regularization introduced by the fact that we, the observers, are like what we are. Although the scheme suggested in 18, 19 is indeed very appealing in its logic, that logic is not necessarily the correct one from the point of view of the observers like ourselves who live and evolve very much along the proper time clocks rather than anything else, and that by itself may be the reason to pick the proper time regularization [4]. Since we ourselves are not gauge invariant objects with respect to time reparametrization, it may be not so bad to have some of the results concerning the probability of creation of domains of our type to be reparametrization dependent too.
The probability distribution $P_{p}(\phi, t)$ used in our previous works has certain advantages as it comes most closely to the description of the total volume of the universe at a given time (including our present time). Therefore we are going to continue its investigation in our subsequent publication [16]. However, it is quite possible that there may be better suggestions. One of the ideas for a physically motivated cut-off can be explained as follows. We speak about the new regions of the universe produced during inflation as of the regions of classical space-time. Meanwhile they are produced by quantum fluctuations. Classical description of the new regions is indeed quite legitimate since they rapidly grow exponentially large. However, they are not infinitely large. There is some time after which the wave function describing different parts of the universe will cease describing classical trajectories and will begin interfere quantum mechanically. We can easily estimate the size of a locally homogeneous part of our universe $\left(\frac{\delta \rho}{\rho} \sim 1\right)$ and the typical time before gravitational collapse of a typical locally Friedmannian part of the universe. This might be the time when the physical cut-off should be introduced. It is not quite clear to us that this is the right way to think about this problem, but it would be very interesting to pursue this idea.

At the present moment we cannot say which of the ways to introduce measure in inflationary cosmology is really preferable. It is possible that eventually we will resolve this problem. Still even that will not guarantee that we are on the right track. Our investigation was based on two hidden assumptions. The first assumption is that we are typical observers. The second assumption is that the number of typical observers is directly proportional to the volume of the universe. If this is correct, then we should live in the place where most observers live, which should correspond to the maximum of the probability distribution.

However, is it not absolutely clear whether the probability for an observer to be born in a particular part of the universe is directly proportional to its volume, or one should take into account something else. One cannot get any crop even from a very large field without having seeds first. The idea that life appears automatically once there is enough space to be populated may be too primitive. It is based on the assumption that one can describe emergence of life solely in terms of physics, and that everything which is necessary for emergence of life was created after inflation. It is certainly the most economical approach, and one should try to go as far as possible without invoking additional hypotheses. One should keep in mind, however, that this approach may happen to be incomplete, especially if consciousness has its own degrees of freedom [1,30]. The nature of the problem can be formulated in purely physical terms as well. Suppose, for example, that emergence of life is catalyzed by the presence of some particles which, like primordial monopoles, cannot be produced at the late stages of in- 
flation. Then the growth of volume of the universe at the late stages of inflation does not increase the total number of such particles and, consequently, the number of observers to be born. In such a situation none of the regularization procedures discussed in the present paper will give a correct information about the parts of the universe where typical observers live.

Another related question is whether we are actually typical? Does it make any sense for each of us to calculate a posteriori the probability to be born Russian, Italian or Chinese? Should we insist on our own mediocrity, or, vice versa, should we try to explain why are we so special? After all, for a long time we thought that we had an aristocratic privilege to be the most intelligent species in the universe. This, of course, may be wrong. Still, before using probabilities to calculate the likelihood of our existence in a particular part of the universe, it may be a good idea to learn more about ourselves. It might happen that until we understand what is our life and what is the nature of consciousness our understanding of quantum cosmology will remain fundamentally incomplete.

In the meantime one may take a pragmatic point of view, postpone answering all these questions and consider our investigation as a kind of "theoretical experiment." We may try to use probabilistic considerations in a trialand-error approach. If we get unreasonable results, this may serve as an indication that we are using quantum cosmology incorrectly. However, if some particular proposal for the probability measure in quantum cosmology will allow us to solve certain problems which could not be solved in any other way, then we will have a reason to believe that this choice of the probability measure is preferable and that perhaps we are moving in the right direction.

We are grateful to A. Vilenkin for many enlightening discussions. This work was supported by NSF grant PHY-8612280.

[1] A. D. Linde, Particle Physics and Inflationary Cosmology (Harwood Academic Press, Chur, Switzerland, 1990).

[2] A. D. Linde, Phys. Lett. 175B, 395 (1986).

[3] A. Vilenkin, Phys. Rev. D 27, 2848 (1983).

[4] A. D. Linde and A. Mezhlumian, Phys. Lett. B 307, 25 (1993); A. D. Linde, D. A. Linde and A. Mezhlumian, Phys. Rev. D 49, 1783 (1994).

[5] A. Linde, Phys. Lett. B 238, 160 (1990); J. GarcíaBellido, A. D. Linde and D. A. Linde, Phys. Rev. D 50, 730 (1994).

[6] J. D. Barrow and F. J. Tipler, The Antropic Cosmological Principle (Clarendon Press, Oxford, 1986).

[7] A. Mezhlumian and A. A. Starobinsky, "Stochastic Inflation: New Results," in: Sakharov Memorial Lectures in Physics, eds. L. V. Keldysh and V. Ya. Fainberg, p.
1119 (Nova Science Publishers, New York, 1992); astro$\mathrm{ph} / 9406045$.

[8] A. D. Linde, Phys. Lett.160B, 243 (1985).

[9] A. D. Linde, Phys. Lett. B 201, 437 (1988); Phys. Lett. B 259, 38 (1991).

[10] A. D. Linde, "Inflation and Quantum Cosmology," in: 300 Years of Gravitation, edited by S.W. Hawking and W. Israel, (Cambridge Univ. Press, Cambridge 1987); A.D. Linde, Phys. Lett. B 227, 352 (1989); A.D. Linde, "Cosmological Constant, Quantum Cosmology and Anthropic Principle," in: Gravitation and Modern Cosmology the Cosmological Constant Problem, eds. A. Zichichi, V. de Sabbata, and N. Sanchez, p. 101 (Plenum Press, New York, 1991).

[11] A. Vilenkin, Phys. Rev. Lett. 74, 846 (1995).

[12] J. García-Bellido and A. D. Linde, Phys. Rev. D 51, 429 (1995).

[13] A. Vilenkin, "Predictions from quantum cosmology," preprint gr-qc/9507018 (1995).

[14] J. García-Bellido and D. Wands, "General Relativity as an Attractor in Scalar-Tensor Stochastic Inflation," SUSSEX-AST-95-3-1 (1995), gr-qc/9503049.

[15] A. D. Linde, D. A. Linde and A. Mezhlumian, Phys. Lett. B 345, 203 (1995).

[16] A. D. Linde, D. A. Linde and A. Mezhlumian, "Nonperturbative Amplification of Inhomogeneities in Stationary Inflation," Stanford University Report No. SUITP-95-25 (in preparation).

[17] J. García-Bellido and A. D. Linde, "Stationary Solutions in Brans-Dicke Stochastic Inflationary Cosmology," Stanford University preprint SU-ITP-95-8 (1995), grqc/9504022, to be published in Phys. Rev. D.

[18] A. Vilenkin, Phys. Rev. D 52, 3365 (1995).

[19] S. Winitzki and A. Vilenkin, "Uncertainties of predictions in models of eternal inflation," Tuft University Report No. TUTP-95-03, gr-qc/9510054.

[20] A. A. Starobinsky, in: Current Topics in Field Theory, Quantum Gravity and Strings, eds. H.J. de Vega and N. Sanchez, Lecture Notes in Physics, vol. 206, p. 107 (Springer, Heidelberg, 1986).

[21] A. S. Goncharov and A. D. Linde, Sov. Phys. JETP 65, 635 (1987); A. S. Goncharov, A. D. Linde and V. F. Mukhanov, Int. J. Mod. Phys. A 2, 561 (1987).

[22] Y. Nambu and M. Sasaki, Phys. Lett. B 219, 240 (1989); Y. Nambu, Prog. Theor. Phys. 81, 1037 (1989).

[23] M. Mijić, Phys. Rev. D 42, 2469 (1990); Int. J. Mod. Phys. A 6, 2685 (1991).

[24] A. Mezhlumian and S. A. Molchanov, J. Stat. Phys., 71, 799 (1993).

[25] A. Mezhlumian, "The Branching Universe", Stanford University Report (in preparation).

[26] M. Aryal and A. Vilenkin, Phys. Lett. B 199, 351 (1987).

[27] G. Paladin and A. Vulpiani, Phys. Rep. 156, 147 (1987).

[28] S. W. Hawking, Phys. Rev. D 32, 2489 (1985).

[29] D. N. Page, Phys. Rev. D 32, 2496 (1985).

[30] D. N. Page, "Sensible quantum mechanics: are only perceptions probabilistic," Preprint of the University of Alberta, ALBERTA-THY-05-95A (1995), quant$\mathrm{ph} / 9506010$. 